Artículo original

(Original paper)

\title{
EFECTO DE LA TEMPERATURA EN POBLACIONES DE DENDROCTONUS FRONTALIS ZIMMERMAN Y DENDROCTONUS MEXICANUS HOPKINS (COLEOPTERA: CURCULIONIDAE: SCOLYTINAE) BAJO UN ESCENARIO DE CAMBIO CLIMÁTICO EN LA SIERRA GORDA QUERETANA
}

\author{
TEMPERATURE EFFECT IN POPULATIONS OF DENDROCTONUS FRONTALIS \\ ZIMMERMAN AND DENDROCTONUS MEXICANUS HOPKINS (COLEOPTERA: \\ CURCULIONIDAE: SCOLYTINAE) UNDER A CLIMATE CHANGE SCENARIO IN SIERRA \\ GORDA QUERETANA
}

\begin{abstract}
ADRIANA MORALES-RANGEL, VÍCTOR HUGo CAMBRÓN-SANDOVAL*, JOSÉ CARMEN SOTO-CORREA, Robert Wallace JONES, JAVIER AlEJANDRo OBREGÓN-ZÚÑIGA

Facultad de Ciencias Naturales. Universidad Autónoma de Querétaro. Av. De las Ciencias S/N, Delegación Santa Rosa Jáuregui, Juriquilla, C. P. 76230 Querétaro, México

<adrianamorales.r@hotmail.com>; <hugo.cambron@gmail.com>; <jocasoco@hotmail.com>; <rjones@uaq.mx>; $<$ jalex.ozuniga.07@gmail.com>

*Autor de correspondencia: <hugo.cambron@gmail.com>
\end{abstract}

Recibido: 23/01/2018; aceptado: 26/06/2018; publicado en línea: 16/11/2018

Editor responsable: Jesús Romero Nápoles

Morales-Rangel, A., Cambrón-Sandoval, V. H., Soto-Correa, J. C., Jones, R.W., Obregón-Zuñiga, J. A. (2018) Efecto de la temperatura en poblaciones de Dendroctonus frontalis Zimmerman y Dendroctonus mexicanus Hopkins (Coleoptera: Curculionidae: Scolytinae) bajo un escenario de cambio climático en la Sierra Gorda queretana. Acta Zoológica Mexicana (nueva serie), 34, 1-8. https://doi.org/10.21829/azm.2018.3412141

RESUMEN. En México fueron afectadas alrededor de 17,900 ha de bosques de coníferas en 2016 a causa de los brotes de insectos descortezadores. Se ha observado que el aumento en las temperaturas ocasionadas por el cambio climático afecta directamente a los bosques de pino y favorece el desarrollo de insectos plaga. Los gradientes altitudinales generan gradientes climáticos, por lo cual, son buenos modelos de estudio en un contexto de cambio climático. El objetivo del estudio fue determinar el efecto de la temperatura y la abundancia de Dendroctonus frontalis y Dendroctonus mexicanus a lo largo del rango de distribución altitudinal, así como determinar un posible cambio en la abundancia para 2030 bajo un escenario de cambio climático. Los resultados evidenciaron que la mayor abundancia de $D$. frontalis y $D$. mexicanus se encuentra en altitudes entre los 1,589 y 1,716 msnm, donde ocurren temperaturas superiores a $16{ }^{\circ} \mathrm{C}$, y en altitudes mayores a los 2,393 msnm la abundancia de ambas especies es menor, correspondiendo a temperaturas menores a $13^{\circ} \mathrm{C}$. El escenario A1B de cambio climático predice que la temperatura aumentará del 2015-2016 al año $20303.9^{\circ} \mathrm{C}$ en promedio.

Palabras clave: Dendroctonus, gradiente altitudinal, temperatura. 
Morales-Rangel, A., Cambrón-Sandoval, V. H., Soto-Correa, J. C., Jones, R.W., Obregón-Zuñiga, J. A. (2018) Temperature effect in populations of Dendroctonus frontalis Zimmerman and Dendroctonus mexicanus Hopkins (Coleoptera: Curculionidae: Scolytinae) under a climate change scenario in Sierra Gorda queretana. Acta Zoológica Mexicana (nueva serie), 34, 1-8. https://doi.org/10.21829/azm.2018.3412141

\begin{abstract}
In Mexico, about 17,900 ha of coniferous forests were affected in 2016 because of outbreaks of bark beetles. The increase in temperatures caused by climate change has been observed that directly affect pine forests and favors the development of insect pests. Altitude gradients generate temperature gradients, which are good study models in a context of climate changes. The aim of this study was to determine the effect of temperature on the abundance of Dendroctonus frontalis and Dendrocotonus mexicanus throughout its altitudinal distribution range, as well as determine a possible future change in abundance by 2030 in a climate change scenario. The results showed the highest abundance of $D$. frontalis and $D$. mexicanus at altitudes between 1,589 to 1,716 masl, where temperatures were higher than $16{ }^{\circ} \mathrm{C}$; however at altitudes upper than 2,393 masl, the abundance of both species were lower, corresponding to temperatures below $13^{\circ} \mathrm{C}$. The A1B climate change scenario predicted that the temperature will increase $3.9^{\circ} \mathrm{C}$ from 2015-2016 to 2030 .
\end{abstract}

Key words: Dendroctonus, altitudinal gradient, temperature.

\title{
INTRODUCCIÓN
}

Los escarabajos descortezadores, entre los que destacan los géneros Dendroctonus, Hylastes e Ips en México, son considerados plagas forestales importantes en bosques de pino (Williams et al., 2008), ya que los ataques masivos de estos insectos provocan la muerte del arbolado, afectando la industria maderera (Atkinson \& Equihua, 1986; Ohmart, 1989; Salinas et al., 2010; Krokene, 2015). El ciclo de vida de los descortezadores depende de su capacidad de vuelo, y del hospedero ideal para iniciar su ciclo. El proceso de colonización comprende de tres etapas principales: la colonización del hospedero, el desarrollo de la cría dentro de los hospederos y la emergencia (Coulson, 1989). Al momento de empezar la colonización, el árbol emite compuestos volátiles (kairomonas) lo cual atrae tanto a hembras como machos hacia el árbol colonizado. Cuando la hembra se une al macho, comienzan la construcción de un sistema de galerías debajo de la corteza donde se desarrollará la progenie (Wood, 1986; Macías-Sámano et al., 2004).

En el año 2016 fueron afectadas alrededor de 17,900 ha de bosques por descortezadores, de las cuales 58.9 ha (0.32\%) correspondieron al estado de Querétaro (CONAFOR, 2016). Las especies de pino más afectadas dentro del estado son Pinus greggii y Pinus patula (Cibrián et al., 2014). Sin embargo, las poblaciones de escarabajos descortezadores también juegan un papel importante en la renovación, saneamiento natural y permanencia de los bosques, en donde las especies los del género Dendroctonus presentan la mayor importancia (Wood, 1982; Raffa et al., 2015). Se ha observado que el crecimiento poblacional de los descortezadores está limitado en ocasiones por variables ambientales como la temperatura y la humedad (Safranyik et al., 2010; Rubín et al., 2015). Según García et al. (2012) la distribución de una especie es modulada de acuerdo a las variaciones climáticas (temperatura, humedad, precipitación, entre otros.) a lo largo de un rango en altitud; así, los gradientes altitudinales son un modelo de estudio adecuado para estimar el cambio climático y distribuciones de especies a futuro (ZacaríasEslava \& Castillo, 2010; Sáenz et al., 2010). Las poblaciones de Dendroctonus dependen directamente de temperaturas elevadas para su desarrollo (Lombardero et al., 2000; Bentz et al., 2014; Six \& Bracewell, 2015) y pueden verse favorecidas por los efectos del calentamiento global, principalmente por el aumento de la temperatura, incrementando su tamaño poblacional al punto de convertirse en plagas forestales (Bentz et al., 2014; Bentz \& Jönson, 2015). Para el año 2030, Soto-Correa et al. (2012) estimaron un aumento de la temperatura en $1.5^{\circ} \mathrm{C}$; aunque, Bentz et al. (2016) predijeron un rango mayor, de 1 a $5{ }^{\circ} \mathrm{C}$. 
El presente estudio se planteó como objetivo, registrar el efecto de la temperatura sobre la abundancia de Dendroctonus frontalis Zimmerman, 1868 y Dendroctonus mexicanus Hopkins, 1909 a lo largo de un gradiente altitudinal dentro de la Sierra Gorda queretana, determinando el cambio en la abundancia de las dos especies de Dendroctonus, en relación al aumento de la temperatura estimada para el año 2030 a lo largo del gradiente altitudinal.

\section{MATERIALES Y MÉTODOS}

Área de estudio. El sistema de muestreo se estableció dentro de bosques de coníferas en dos localidades al norte del estado de Querétaro: La Pingüica (2109'25.99” N, 9941’44.00” O) en el municipio de Pinal de Amoles y El Lobo (21¹7'34.00” N, 9907’09.99” O) en el municipio de Landa de Matamoros (INEGI, 2016). Las especies de coníferas presentes en los sitios de estudio fueron Pinus patula Schiede ex Schltdl \& Cham., P. montezumae Lamb., P. ayacahuite Ehrenb. Ex Schltdl., P. teocote Schiede ex Schltdl. \& Cham., P. greggii Engelm. Ex Parl., P. rudis, P. cembroides y P. pinceana Gordon \& Glend.; así como algunas especies del género Quercus, dentro de las que destacan: Q. crassifolia Bonpl. y Q. greggii (A.DC.) Trel. (en altitudes entre 2,200 a 3,100 msnm), Q. mexicana Humb. \& Bonpl. y Q. castanea Née (entre los 1,200 a 2,300 msnm) (Carabias et al., 1999). Los sitios se caracterizaron por un clima templado húmedo a semicálido sub-húmedo con lluvias en verano y una precipitación media anual de 850-920 mm y una temperatura media anual de $12-24{ }^{\circ} \mathrm{C}$. La temperatura máxima se presentó en abril y mayo y la mínima en los meses de diciembre y enero (INEGI, 2009).

La selección de sitios se realizó dentro de un gradiente altitudinal en intervalos de 100 m (MacíasSámano \& Niño-Domínguez, 2016). El gradiente altitudinal fue de los 1,589 a los 3,058 msnm. Dentro de la localidad de Pinal de Amoles se situaron ocho sitios de muestreo desde los 2,393 a los 3,058 msnm. Mientras que en la localidad Landa de Matamoros se situaron cuatro sitios de muestreo entre los 1,589 a 1,716 msnm (Cuadro 1).

Cuadro 1. Sitios de muestreo de las especies de $D$. frontalis y $D$. mexicanus, abundancia, temperaturas ocurridas entre los años 2015-2016 y estimación al año 2030.

\begin{tabular}{|c|c|c|c|c|c|c|c|}
\hline \multirow{2}{*}{ Nom } & \multirow{2}{*}{$\begin{array}{l}\text { Altitud } \\
\text { (msnm) }\end{array}$} & \multirow{2}{*}{ Lat $\mathbf{N}$} & \multicolumn{2}{|c|}{ Abundancia } & \multicolumn{2}{|c|}{$\begin{array}{c}\text { Temperatura promedio } \\
\left({ }^{\circ} \mathrm{C}\right)\end{array}$} & \multirow{2}{*}{$\begin{array}{l}\text { Diferencia d } \\
\text { temperatura } \\
\quad\left({ }^{\circ} \mathrm{C}\right)\end{array}$} \\
\hline & & & D. fronta & D. mexicanus & $2015-2016$ & 2030 & \\
\hline Lob 1 & 1589 & 9907'25.60080" 21ํ1'51.79920" & 14544 & 1634 & 18 & 21.4 & 3.4 \\
\hline Lob 2 & 1651 & 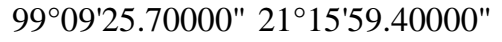 & 19897 & 3418 & 16.63 & 21.2 & 4.57 \\
\hline Mad 1 & 1670 & 9907'23.49998" 21ำ1'51.10001" & 9122 & 984 & 16.51 & 21.1 & 4.59 \\
\hline Mad 2 & 1716 & 9908'55.60001" 21ำ16'54.59999" & 38234 & 1244 & 16.89 & 21 & 4.11 \\
\hline Pin 1 & 2393 & $99^{\circ} 37^{\prime} 35.90000^{\prime \prime} 21^{\circ} 07^{\prime} 54.99998^{\prime \prime}$ & 303 & 93 & 13.66 & 17.8 & 4.14 \\
\hline Pin 2 & 2468 & $99^{\circ} 38^{\prime} 00.10000^{\prime \prime} 21^{\circ} 07^{\prime} 48.29999^{\prime \prime}$ & 274 & 146 & 12.79 & 17.5 & 4.71 \\
\hline Pin 3 & 2541 & $99^{\circ} 39^{\prime} 50.00000^{\prime \prime} 21^{\circ} 07^{\prime} 42.20000^{\prime \prime}$ & 182 & 138 & 12.21 & 17.1 & 4.89 \\
\hline Pin 4 & 2610 & $99^{\circ} 40^{\prime 29.20001 " 21^{\circ} 07 ' 18.00001 "}$ & 296 & 187 & 13.06 & 16.7 & 3.64 \\
\hline Pin 5 & 2706 & 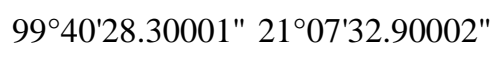 & 721 & 232 & 13.51 & 16.2 & 2.69 \\
\hline Pin 6 & 2790 & $99^{\circ} 40^{\prime 39.20002 " ~ 21^{\circ} 07 ' 31.19999 "}$ & 709 & 167 & 12.54 & 15.7 & 3.16 \\
\hline Pin 7 & 2904 & 9940'55.99999" 2107'28.49999" & 541 & 415 & 11.57 & 15.1 & 3.53 \\
\hline Pin 8 & 3058 & 9941'36.20000" 2109'42.19999" & 312 & 67 & 10.77 & 14.2 & 3.43 \\
\hline
\end{tabular}


Establecimiento de trampas. En cada sitio de muestreo se colocaron trampas tipo Lindgren de ocho embudos con vaso colector por duplicado (Synergy Semiochemicals Corps ${ }^{\circledR}$ ) separadas 50 metros longitudinalmente una de otra; dichas trampas se instalaron en árboles no hospederos, quedando suspendidas a $1.5 \mathrm{~m}$ del suelo; una trampa fue cebada con una combinación de semioquímicos (SQ's) para Dendroctonus spp. de Synergy Semiochemicals Corps ${ }^{\circledR}$ (Macías-Sámano y Niño, 2016), con las feromonas Frontalina + endobrevicomina y alfa/beta pineno como kairomona; la otra trampa se estableció como trampa testigo sin feromonas. En el vaso colector de cada trampa se colocó anticongelante Bardahl ${ }^{\circledR}$ para motor (Etilenglicol [30-40\%], aditivos inhibidores de corrosión [1-2\%], Agua [60-70\%]) y alcohol al $70^{\circ}$ en concentración 1:1, para facilitar sacrificio y la conservación de los insectos (Zylstra et al., 2010).

Colecta e identificación de insectos. La colecta de los insectos se llevó a cabo durante el periodo de abril 2015 a marzo 2016. Cada 15 días se colectaron los insectos atrapados en el vaso colector depositándolos en bolsas tipo Ziploc ${ }^{\circledR}$. Posteriormente se procedió a limpiar y separar los individuos descortezadores de otros grupos de insectos. La identificación de las especies del género Dendroctonus se realizó con la ayuda de las claves taxonómicas de Wood (1982) y Cibrián et al. (1995).

Temperatura. El monitoreo de la temperatura se realizó mediante recolectores de datos (EL-USB-2 RH/data logger de Micro DAQ ${ }^{\circledR}$ ) durante abril 2015 a marzo 2016, los cuales tomaron una lectura cada 30 minutos, se colocaron en cada sitio de muestreo entre cada par de trampas (Cuadro 1). Los datos de temperatura predichos para el 2030 se estimaron por un escenario intermedio A1B (modelo intermedio; A: altas emisiones y B: bajas emisiones de carbono) (Crookston, 2010) (Cuadro 1). De cada punto seleccionado se estimaron las variables climáticas de temperatura media anual contemporáneos (19611991) y futuros 2030 A1B (Crookston, 2010 y 2014); dichos valores se calcularon de acuerdo a estimaciones del Panel Intergubernamental de Cambio Climático (IPCC) y posteriormente ajustando el modelo en ANUSPLINE (Sáenz-Romero et al., 2010; Crookstron, 2010). Las variables climáticas se obtuvieron mediante el modelo climático "thin plate spline”, desarrollados para México (Crookston, 2010), el cual se basa en la transposición de superficies de datos climáticos mensuales normalizados de temperatura (mínima, media y máxima) y precipitación, colectados de aproximadamente 4000 estaciones climatológicas en el sur de Estados Unidos, México, Guatemala, Honduras y Cuba.

Análisis de datos. Se realizó un análisis de varianza (ANOVA) y una prueba múltiple de medias de Tukey $(\alpha=0.05)$ con el paquete estadístico SAS (versión 9.3 de SAS Institute) para denotar posibles diferencias significativas en la abundancia de $D$. frontalis y $D$. mexicanus, y comparación entre medias. La regresión se realizó para observar la relación de variables temperatura-altitud, así como para estimar el grado de inferencia entre ambas.

\section{RESULTADOS Y DISCUSIÓN}

Abundancias de $\boldsymbol{D}$. frontalis y $\boldsymbol{D}$. mexicanus. Se estimaron diferencias significativas entre las abundancias a diferentes altitudes de $D$. frontalis (ANOVA: $N=177, G l=11, F=13.48, P=0.0001$ ) y $D$. mexicanus (ANOVA: $N=166, G l=11, F=5.76, P=0.0001$ ), donde se observó que la mayor abundancia de $D$. frontalis y $D$. mexicanus está en altitudes entre los 1,589 y 1,716 msnm, con abundancias totales de entre 38,234 y 9,122 individuos respectivamente para $D$. frontalis, en comparación con $D$. mexicanus con abundancias totales entre 984 a 1,634 individuos, respectivamente (Cuadro 1). Este gran número de individuos sugiere un ataque masivo, que generalmente termina en la muerte del arbolado (Goodsman et al., 2016). Estas abundancias ocurrieron a temperaturas medias anuales registradas por los Data logger durante abril 2015 a marzo 2016 que oscilaron entre 16.63 y $18{ }^{\circ} \mathrm{C}$ para el presente estudio; sin embargo, en otros estudios se conoce que para Dendroctonus ponderosae la temperatura óptima es mayor a $15^{\circ} \mathrm{C}$ (Bentz et al., 2014). 
El análisis de las pruebas de medias de Tukey se observan dos grupos; en el primero, las mayores abundancias al igual que las temperaturas mayores $\left(16\right.$ y $\left.18{ }^{\circ} \mathrm{C}\right)$, presentaron los mismos integrantes, esto ocurrió en altitudes menores entre 1,589 y 1,716 msnm (Cuadro 2); en otros estudios se ha observado que la abundancia de descortezadores aumenta en menores altitudes (Tykarski, 2006). Las temperaturas medias anuales entre 16 y $18{ }^{\circ} \mathrm{C}$ son promotoras de una mayor abundancia, esto es similar a lo observado por Six y Bracewell (2015) en el género Dendroctonus y en D. ponderosae por Bentz et al. (2014). El segundo grupo en la prueba de medias de Tukey, presentó abundancias menores entre los $721\left(13.51^{\circ} \mathrm{C}\right)$ y $182\left(12.21^{\circ} \mathrm{C}\right)$ individuos para $D$. frontalis, mientras que para $D$. mexicanus se observó una abundancia entre los 146 y 67 individuos, esto ocurrió en altitudes de 2,393 a 3,058 msnm donde ocurrieron temperaturas entre 12.79 y $10.77^{\circ} \mathrm{C}$ coincidiendo los integrantes de ambos grupos (Cuadro 2). Lo anterior es evidencia de que una menor abundancia $D$. frontalis y $D$. mexicanus es promovida por temperaturas medias anuales por debajo de los trece grados; autores como Ungerer et al. (1999), Logan y Powell (2001) y Six y Bracewell (2015) lo comprueban al observar que la abundancia de Dendroctonus disminuye al presentarse temperaturas muy bajas.

Cuadro 2. Prueba de Tukey de abundancia de $D$. frontalis y $D$. mexicanus y temperaturas ocurridas entre 2015-2016 y estimadas al 2030 en un escenario A1B.

\begin{tabular}{cccccc}
\hline Trampa & Nombre & $\begin{array}{c}\text { Altitud } \\
(\mathrm{m})\end{array}$ & $\begin{array}{c}\text { D. frontalis } \\
\text { Media }\end{array}$ & $\begin{array}{c}\text { D. mexicanus } \\
\text { Media }\end{array}$ & $\begin{array}{c}\text { Temperatura }\left({ }^{\circ} \mathbf{C}\right) \\
\text { Media }\end{array}$ \\
\hline 1 & Lobo1 & 1589 & $692.6 b c$ & $77.81 a b$ & $15.97 a$ \\
2 & Lobo2 & 1651 & $904.4 b$ & $155.36 a$ & $15.97 a$ \\
3 & Madroño1 & 1670 & $396.6 b c$ & $42.78 b$ & $15.58 a$ \\
4 & Madroño2 & 1716 & $1737.9 a$ & $56.55 a b$ & $15.56 a$ \\
5 & Pingüica 1 & 2393 & $27.5 c$ & $10.33 b$ & $12.83 b$ \\
6 & Pingüica 2 & 2468 & $24.9 c$ & $12.17 b$ & $11.97 b c$ \\
7 & Pingüica 3 & 2541 & $14 c$ & $12.55 b$ & $11.46 b c$ \\
8 & Pingüica 4 & 2610 & $21.1 c$ & $15.58 b$ & $12.36 b$ \\
9 & Pingüica 5 & 2706 & $65.5 c$ & $25.78 b$ & $12.54 b$ \\
10 & Pingüica 6 & 2790 & $64.5 c$ & $18.56 b$ & $10.88 b c$ \\
11 & Pingüica 7 & 2904 & $45.1 c$ & $37.73 b$ & $10.88 b c$ \\
12 & Pingüica 8 & 3058 & $52 c$ & $13.4 b$ & $10.04 c$ \\
\hline \multicolumn{7}{c}{ Nota: letras diferentes significan diferencias significativas de P $>0.05$}
\end{tabular}

Se estimó una relación positiva entre la temperatura media anual ocurrida en el periodo de abril 2015 a marzo 2016 respecto a la altitud $\left(r^{2}=0.5, p=0.0001\right)$, donde a mayor altitud la temperatura media anual disminuye, lo mismo ocurre con la estimación al año 2030 escenario intermedio A1B, el cual tuvo una relación superior con la altitud $\left(r^{2}=0.9974, p=0.0001\right)$ (Fig. 1). Comparando los datos de temperatura registrados por los Data logger y los estimados para el año 2030 se predice un aumento en la temperatura media anual en promedio de todos los sitios de $3.9{ }^{\circ} \mathrm{C}$, lo cual parece muy elevado, aunque en comparación con otros trabajos se ha estimado un aumento de la temperatura media anual de aproximadamente $1.5^{\circ} \mathrm{C}$ (Soto-Correa et al., 2012), aunque Bentz et al. (2016) predice un incremento en la temperatura de 1 a $5{ }^{\circ} \mathrm{C}$ en diferentes escenarios; de tal manera que con el aumento en la temperatura se ha observado que favorece el crecimiento poblacional de los descortezadores (Bentz \& Jönson, 2015). Analizando un cambio menor dentro de los rangos de temperatura, el sitio donde ocurre el menor cambio al año 2030, el aumento de la temperatura es de $2.69^{\circ} \mathrm{C}$, esto ocasionaría que en sitios a mayor elevación para el año 2030 ocurran temperaturas por encima de $16^{\circ} \mathrm{C}$ en altitudes hasta 2,700 msnm donde ahora se presentan temperaturas menores de $13{ }^{\circ} \mathrm{C}$ correspondientes a menores abundancias de insectos 
descortezadores (Fig. 1). Si se considera que las mayores abundancias están asociadas a temperaturas medias anuales por encima de los $16{ }^{\circ} \mathrm{C}$, en el año 2030 con el aumento de temperatura se esperaría que la abundancia de $D$. frontalis y $D$. mexicanus aumente en sitios con elevaciones hasta los 2,700 msnm donde hoy existe poca abundancia.

\section{Conclusión}

El estudio demuestra que, en el rango de distribución altitudinal en la Sierra Gorda queretana, Dendroctonus frontalis y Dendroctonus mexicanus presentan mayor abundancia en temperaturas mayores de $16{ }^{\circ} \mathrm{C}$ que ocurren a altitudes por debajo de los $1,716 \mathrm{msnm}$ y menores abundancias en temperaturas por debajo de $13{ }^{\circ} \mathrm{C}$ con altitudes superiores a 2,393 msnm. Las estimaciones de temperatura en escenario de cambio climático al 2030 mostró un aumento en promedio de $3.9^{\circ} \mathrm{C}$ en todos los sitios, por lo que se puede predecir que de continuar con las tendencias actuales en el manejo de la contaminación y deforestación, se presenten temperaturas de $16{ }^{\circ} \mathrm{C}$ en altitudes hasta los $2,700 \mathrm{~m}$ y como consecuencia incremento de las poblaciones de $D$. frontalis y $D$. mexicanus.

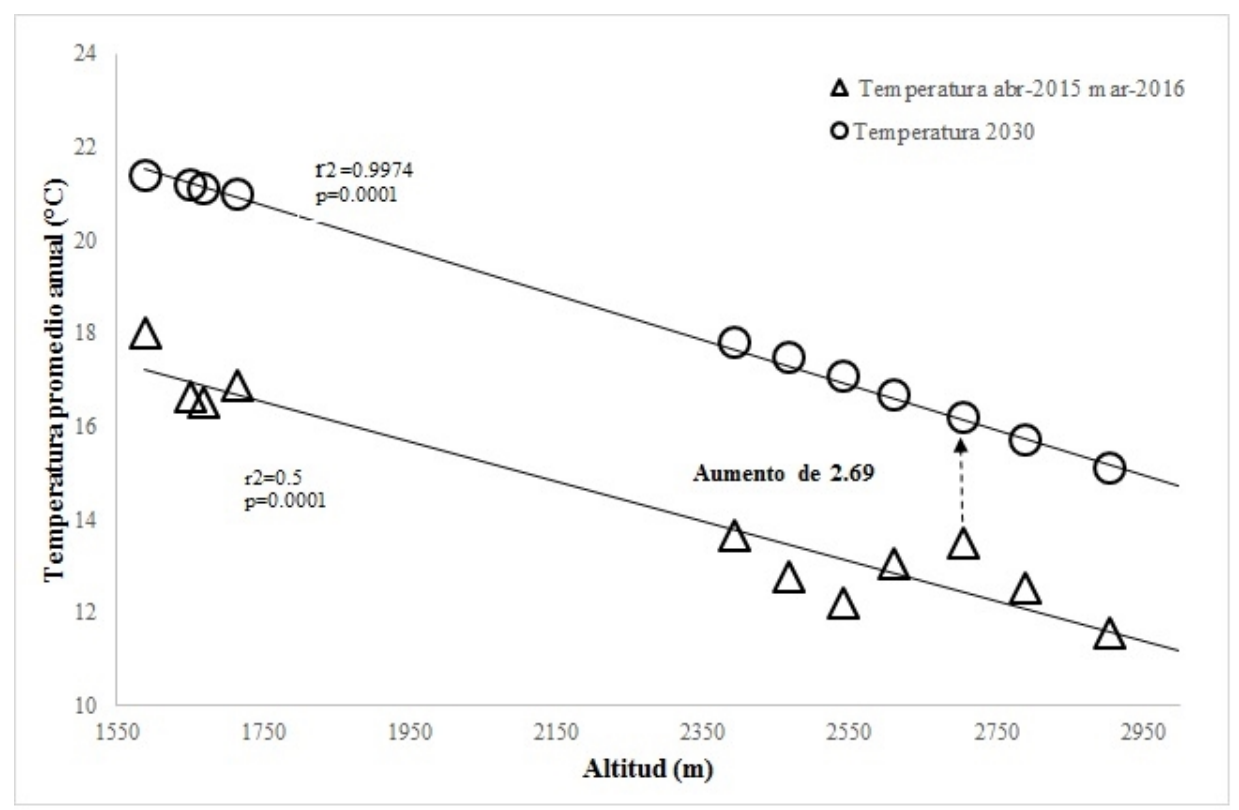

Figura 1. Temperatura actual del periodo 2015 a 2016 y estimación de temperatura del escenario A1B al año 2030.

Agradecimientos. Al fondo CONAFOR-CONACyT C01-234547 por el apoyo brindado para la realización del proyecto.

\section{LITERATURA CITADA}

Atkinson, T. H., Equihua, A. (1986) Biology of the Scolytidae and Platypodidae (Coleoptera) in a tropical deciduous forest at Chamela, Jalisco, Mexico. Florida Entomologist, 69(2), 303-310.

Bentz, B., Jönsson, A. M. (2015) Modeling bark beetles response to climate change. Pp. 533-553. In: Bark beetles: biology and ecology of native and invasive species. Academic Press 
Bentz, B., Vandygriff, J., Jensen, C., Coleman, T., Maloney, P., Smith, S., Schen-Langenheim, G. (2014) Mountain pine beetle voltinism and life history characteristics across latitudinal and elevational gradients in the western United States. Forest Science, 60(3), 434-449.

Bentz, B. J., Duncan, J. P., Powell, J. A. (2016) Elevational shifts in thermal suitability for mountain pine beetle population growth in a changing climate. Forestry, 89(3), 271-283.

Carabias Lillo, J., Provencio, E., de la Maza Elvira, J., Ruiz Corzo, M. (1999) Programa de Manejo Reserva de la Biosfera Sierra Gorda. México, Instituto Nacional de Ecología, SEMARNAT (Secretaria de Medio Ambiente y Recursos Naturales).

Cibrián Tovar, D., Arango Caballero, L., Tovar, D. C. (1995) Insectos forestales de México (No. SB764. M6, 15). Universidad Autónoma de Chapingo.

Cibrián Tovar, D., Quiñonez, S. A., Morales, F., J., Ortiz, B. O., Bocanegra H., S., Torres, F. U., Santiago, G. E., Macías, S. H. J., Curiel, C. M., Cortés, E. E. (2014) Diagnóstico de situación actual y monitoreo de insectos descortezadores en los bosques templados de la reserva de la Biósfera sierra Gorda (RBSG), Universidad Autónoma de Chapingo (UACH), comisión Nacional de Áreas Naturales Protegidas (CONANP), Comisión Nacional forestal (CONAFOR) y Deutsche Gesellschaft für Internationale zusammenarbeit (GIZ) GmbH. México.

CONAFOR (Comisión Nacional Forestal) (2016) Alerta temprana y evaluación de riesgo para insectos descortezadores Diciembre 2016.

Coulson, R. N., Feldman, R. M., Sharpe, P. J. H., Pulley, P. E., Wagner, T. L., Payne, T. L. (1989) An overview of the TAMBEETLE model of Dendroctonus frontalis population dynamics. Ecography, 12(4), 445-450.

Crookston, N. L. (2010) Research on Forest Climate Change: Potential effects of Global Warming on Forest and Plant Climate Relationship in Western North America and Mexico http://forest.moscowfsl.wsu.edu/climate/ (Consultado: Febrero 2017).

García López, A., Micó, E., Galante, E. (2012) From lowlands to highlands: searching for elevational patterns of species richness and distribution of scarab beetles in Costa Rica. Diversity and Distributions, 18(6), 543-553.

Goodsman, D. W., Koch, D., Whitehouse, C., Evenden, M. L., Cooke, B. J., Lewis, M. A. (2016) Aggregation and a strong Allee effect in a cooperative outbreak insect. Ecological Applications, 26(8), 2621-2634.

INEGI (Instituto Nacional de $\quad$ Estadística $y \quad$ Geografía). http://www3.inegi.org.mx/sistemas/mexicocifras/datos-geograficos/22/22002.pdf

INEGI (Instituto Nacional de Estadística y Geografía) (2016) http://www.inegi.org.mx/. Fecha de consulta: Octubre 2016.

Krokene, P. (2015) Conifer defense and resistance to bark beetles. Pp. 177-207 In: Bark beetles: biology and ecology of native and invasive species, Academic Press.

Logan, J. A., Powell, J. A. (2001) Ghost forest, global warming, and the mountain pine beetles (Coleoptera; Scolytidae). American Entomologist, 47(3), 160.

Lombardero, M. J., Ayres, M. P., Ayres, B. D., Reeve, J. D. (2000) Cold tolerance of four species of bark beetle (Coleoptera: Scolytidae) in North America. Environmental Entomology, 29(3), 421-432.

Macías Sámano, J. E., Domínguez A. N., López, J. C., Mérida, R. A. (2004) Monitoreo de descortezadores y sus depredadores mediante el uso de semioquímicos: Manual operativo. EcosurConafor.CONANP-USDA Forest Service. Tapachula, Chiapas, Méx. Pp. 11.

Macías Sámano, J. E., Niño Domínguez, A. (2016) Protocolo para monitoreo de descortezadores de coníferas mediante el uso de atrayentes y semioquímicos para México y Centroamérica. Colegio de la Frontera Sur, San Cristóbal de las Casas, Chiapas, México. Pp.14.

Ohmart, C. P. (1989) Why are there so few tree-killing bark beetles associated with angiosperms. Oikos, 242-245.

Raffa, K. F., Gregoire, J. C., Lindgren, B. S. (2015) Natural history and ecology of bark beetles Pp. 1-40. In: Bark beetles: Biology and Ecology of Native and Invasive Species. Academic Press. 
Rubín Aguirre, A., Sáenz-Romero, C., Lindig-Cisneros, R., del Río Mora, A. A., Tena Morelos, C. A., Campos Bolaños, R., del Val, E. (2015) Bark beetle pests in an altitudinal gradient of a Mexican managed forest. Forest Ecology and Management, 343, 73-79.

Sáenz Romero, C., Rehfeldt, G. E., Crookston, N. L., Duval, P., St. Amant, R., Beaulieu, J., Richardson, B. A. (2010) Spline models of contemporary, 2030, 2060 and 2090 climates of Mexico and their use in understanding climate-change impacts on the vegetation. Climate Change, 102(3), 595-623.

Safranyik, L., Carrol, A. L., Régniére, J., Langor, D. W., Riel, W. G., Shore, T. L., Taylor, S. W. (2010) Potentitial for range expansion of mountain pine beetle into the boreal forest of North America. The Canadian Entomologist, 142(5), 415-442.

Salinas Moreno, Y., Vargas, M. C. F., Zúñiga, G., Víctor, J., Ager, A., Hayes, J. L. (2010) Atlas de distribución geográfica de los descortezadores del género Dendrcotonus (Curculionidae: Scolytinae) en México. Instituto Politécnico Nacional, Comisión Nacional Forestal, México, DF. Pp. 31, 35.

Six, D. L., Bracewell, R. R. (2015) Dendroctonus. Pp. 305-350. In: Bark beetles: Biology and Ecology of Native and Invasive Species. Academic Press.

Soto-Correa, J. C., Sáenz-Romero, C., Lindig-Cisneros, R., Sánchez Vargas, N., Cruz de León, J. (2012) Variación genética entre procedencias de Lupinus elegans Kunth, zonificación altitudinal y migración asistida. Agrociencia, 46(6), 593-608.

Tykarski, P. (2006) Beetles associated with scolytids (Coleoptera: Scolytidae) and the elevational gradient: Diversity and dynamics of the community in the Tatra National Park, Poland. Forest ecology and management, 225(1), 146-159.

Ungerer, M. J., Ayres, M. P., Lombardero, M. J. (1999) Climate and the northern distribution limits of Dendroctonus frontalis Zimmermann (Coleoptera: Scolytidae). Journal of Biogeography, 26(6), 1133-1145.

Williams, K., McMillin, J. D., DeGomez, T. E., Clancy, K. M., Anhold, J. A., Miller, A. (2008) Influence of elevation on bark beetle community structure in ponderosa pine stands of north-central Arizona. Environmental Entomology, 37, 94-109.

Wood, S. L. (1982) The bark and ambrosia beetles of North and Central America (Coleoptera: Scolytidae), a taxonomic monograph. Brigham Young University, Provo, UT,

Zacarías Eslava, Y., Castillo, R. F. D. (2010) Comunidades vegetales templadas de la Sierra Juárez, Oaxaca: pisos altitudinales y sus posibles implicaciones ante el cambio climático. Boletín de la sociedad Botánica de México, (87), 13-28.

Zylstra, K. E., Dodds, K. J., Francese, J. A., Mastro, V. (2010) Sirex noctilio in North America: the effect of stem-injection timingon the attractivenessand suitability of trap tres. Agricultural and Forest Entomology, 12(3), 243-250. 